MAGNET CURRENT REGULATION IN THE SSC

O. Calvo, G. Tool, and D. Wolff

April 1986 
TM-1397

0102.000

SSC-N-161

\title{
Magnet Current Regulation in the SSC
}

\author{
O. Calvo, G. Tool, D. Wolff \\ April, 1986
}

\begin{abstract}
This paper investigates the transient response, the stability and the regulation characteristics of a system designed to power the SSC magnets. Considering the magnet system as a transmission line, the performance of the regulation system was investigated under perturbations on the voltage power supply and changes in the current reference. The influence of the damping resistors on the transient response was included.

Differential current transductors were included to minimize the tracking errors between the electrically isolated sectors. A comparision was made for the two cases, with and without the differential loops. System performance was investigated during ramping of the magnets and during steady state full field operation.
\end{abstract}

\section{Introduction}

Practical considerations in the preliminary design of the SSC power distribution system have shown the desirability of electrically isolating the magnet circuits into ten separate sectors. Preliminary analysis of the SSC, discussed in the same reference, has indicated that the tracking between the quadrupoles and dipole magnets and from one sector to another, must be to a few parts per $10^{5}$. The objective of this paper is to present a possible scheme for the current regulation that is capable of achieving these requirements in a relatively simple manner. Before determining the performance of the whole system, it is necessary to analyze an individual sector.

\section{Closed Loop Response of the Individual Sectors}

The regulation and control system consists of two major loops; an internal one controlling the power supply voltage and an external one monitoring the current in the magnets. The voltage loop is designed to have a high frequency response and its main function is to regulate the voltage applied to the magnets. It must follow a predetermined voltage reference related to the current reference, rejecting line voltage changes, load changes, offset errors and other perturbations. The external loop is the magnet current regulator. It has relatively low frequency response and very high gain in order to meet the dc accuracy requirements.

1. "Conceptual Design Superconducting Super Collider", SSC Central Design Group, March, 1986 


\section{Voltage Loop}

The power supply consists of a twelve pulse rectifier, a firing pulse generator, a linearization circuit, a voltage controller and a feedback circuit. A block diagram of the power supply including a passive filter is shown in figure 1.

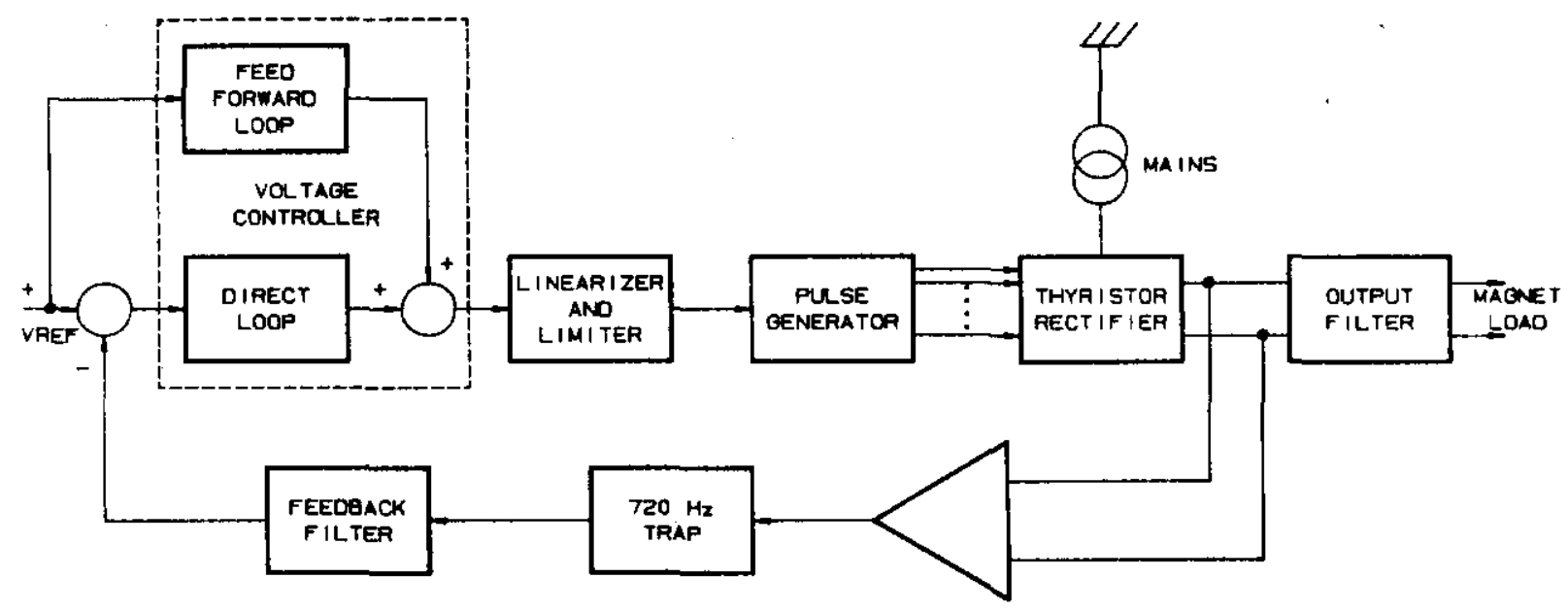

Figure 1. Power Supply Voltage Regulator Block Diagram

An accurate model for the power supply was developed using ACSL. ${ }^{2}$ The model includes phase overlap effects, characteristic ripple components, noncharacteristic subharmonic contents, quantification errors, saturation elements and frequency bandwith of amplifiers, etc. (A complete description of the model can be found in a separate paper. $)^{3}$ In order to reduce the ripple at the output of the power supply, an output filter consisting of a second order filter with a roll-off frequency of $60 \mathrm{~Hz}$ and a $720 \mathrm{~Hz}$ trap was included.

The circuit used is a typical one for this type of magnet power supply. 4 As the power supply used in the model was very similar to the one used in the Energy Saver at Fermilab, the results for a step of the voltage reference were verified against measured values obtained under the same circumstances.

\section{Current Loop}

The current regulator loop of an individual sector is composed of the following elements: power supply with external voltage reference, string of magnets considering transmission line effects, dc current transductor, digital current reference from a host computer and current controller. A block diagram is shown in figure 2.

2. ACSL is an Advanced Continuous Simulation Language developed by Mitchell and Gauthier, Assoc., to model and evaluate the performance of continuous systems described by time dependent, non linear differential equations.

3. Simulation of the Energy Saver SCR Power Supply Using ACSL, Fermilab Accelerator Division Internal Note.

4. Fermilab Energy Saver Power Supply Design Notes, Dan Wolff 


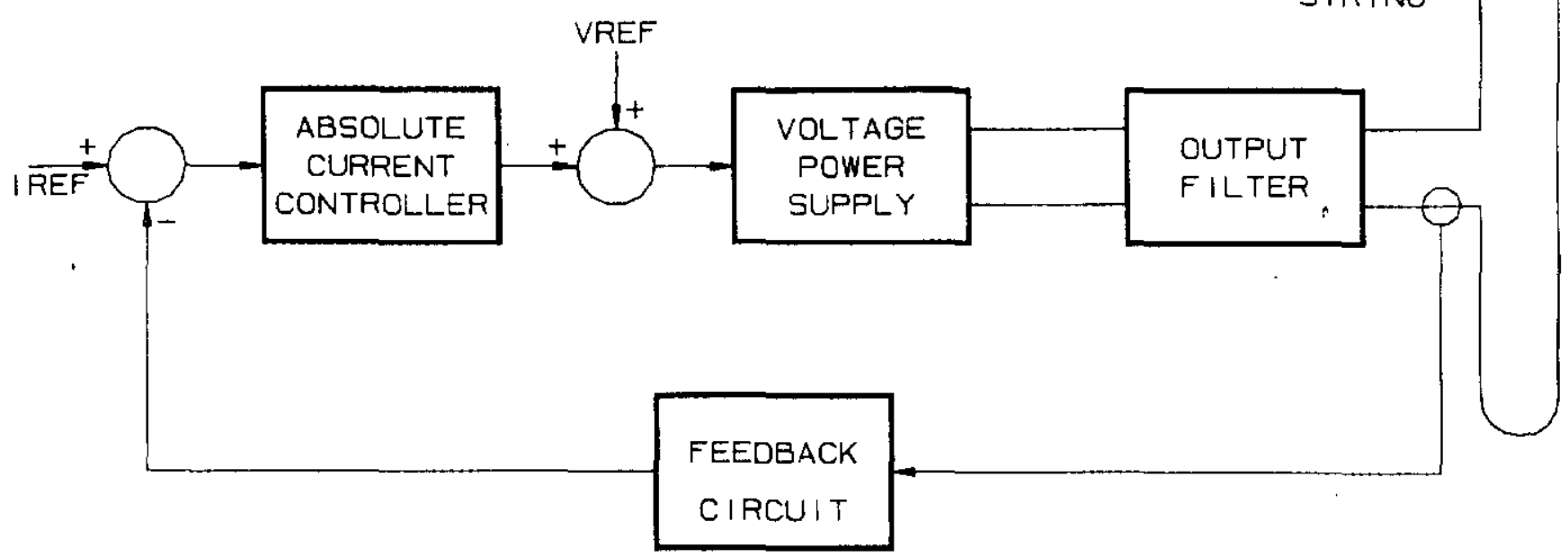

Figure 2. Sector Current Regulator Block Diagram

The program SPICE was used in this case to simulate the current loop to provide further insight into the behaviour of the magnet string. ${ }^{2}$ The reference sector is assumed to contain 80 half-cells of 5 dipoles each, or 400 dipoles total. Eddy current effects, modeled as a fixed resistance, and an external damping resistor of 25 ohms/dipole were included in the model. The SPICE model for one sector is shown in figure 3.

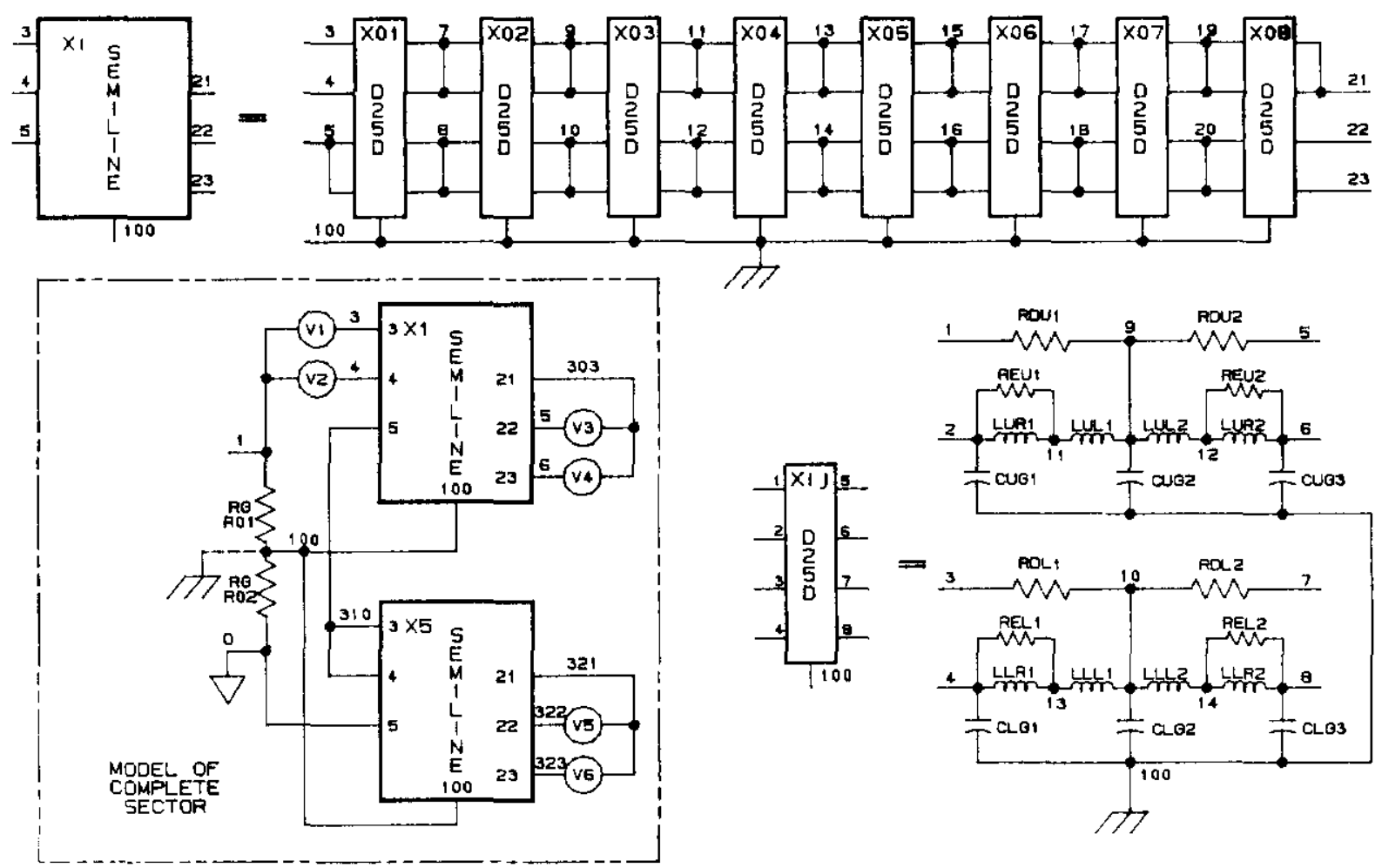

Figure 3. SPICE Model for One Sector of SSC Magnets

5. SPICE is a general purpose circuit simulation program developed by the Department of Electrical Engineering and Computer Sciencies at University of California, Berkeley and used throughout the electronics industry. 
The remaining elements of the system were simulated using operational amplifiers connected in the appropriate configuration to obtain the desired transfer functions as shown in figure 4.

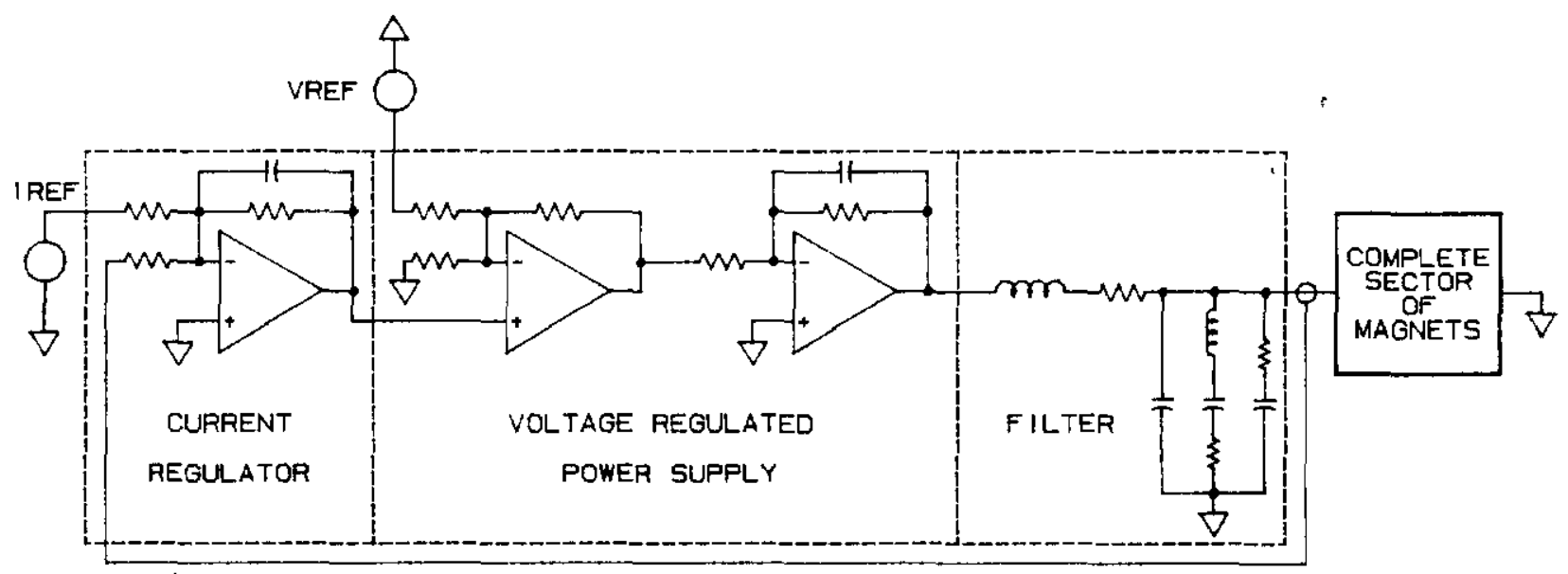

Figure 4. SPICE Model of Current Regulation Loop of One Sector

Due to the inherent low pass characteristic of the magnet string the voltage power supply model developed with ACSL was replaced by a first order system, without significantly affecting the accuracy of the current response of this loop. The transfer function of this model is shown in figure 5.

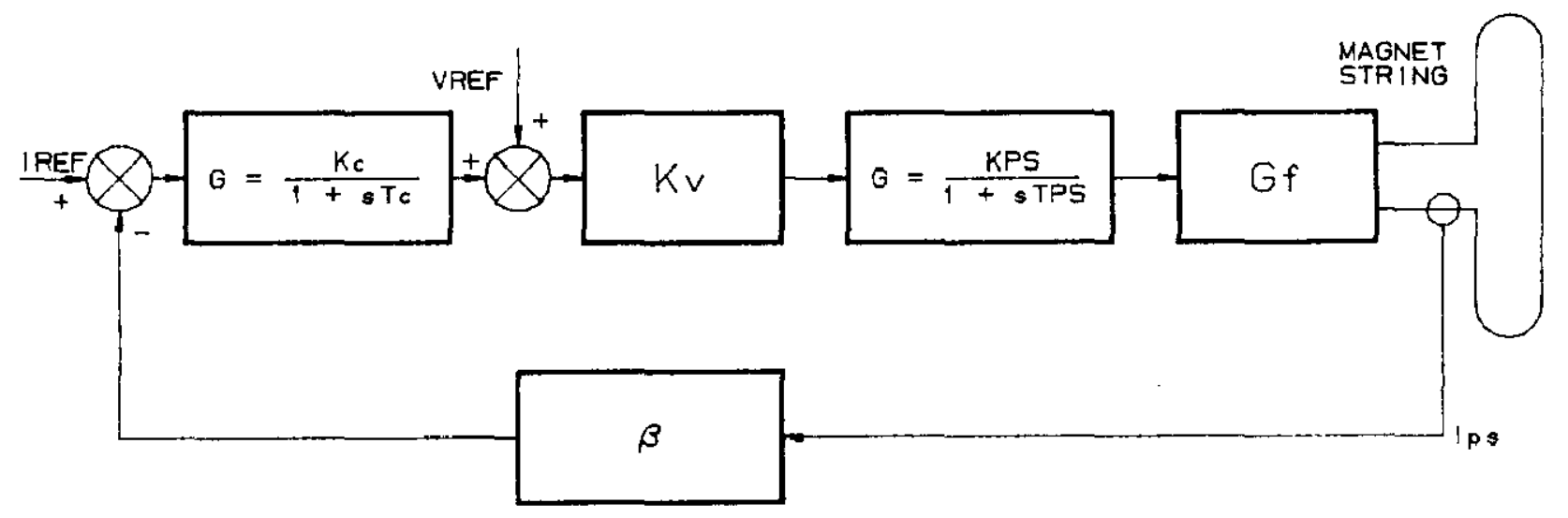

Figure 5. Transfer Function Model of One Sector

The frequency response of this circuit for a dc gain of 130,000 is shown in figure 6. It can be seen that the system is stable with a crossover frequency close to $9 \mathrm{~Hz}$. A more careful design of this loop may lead to improved bandwidth. The present circuit values are very conservative. 


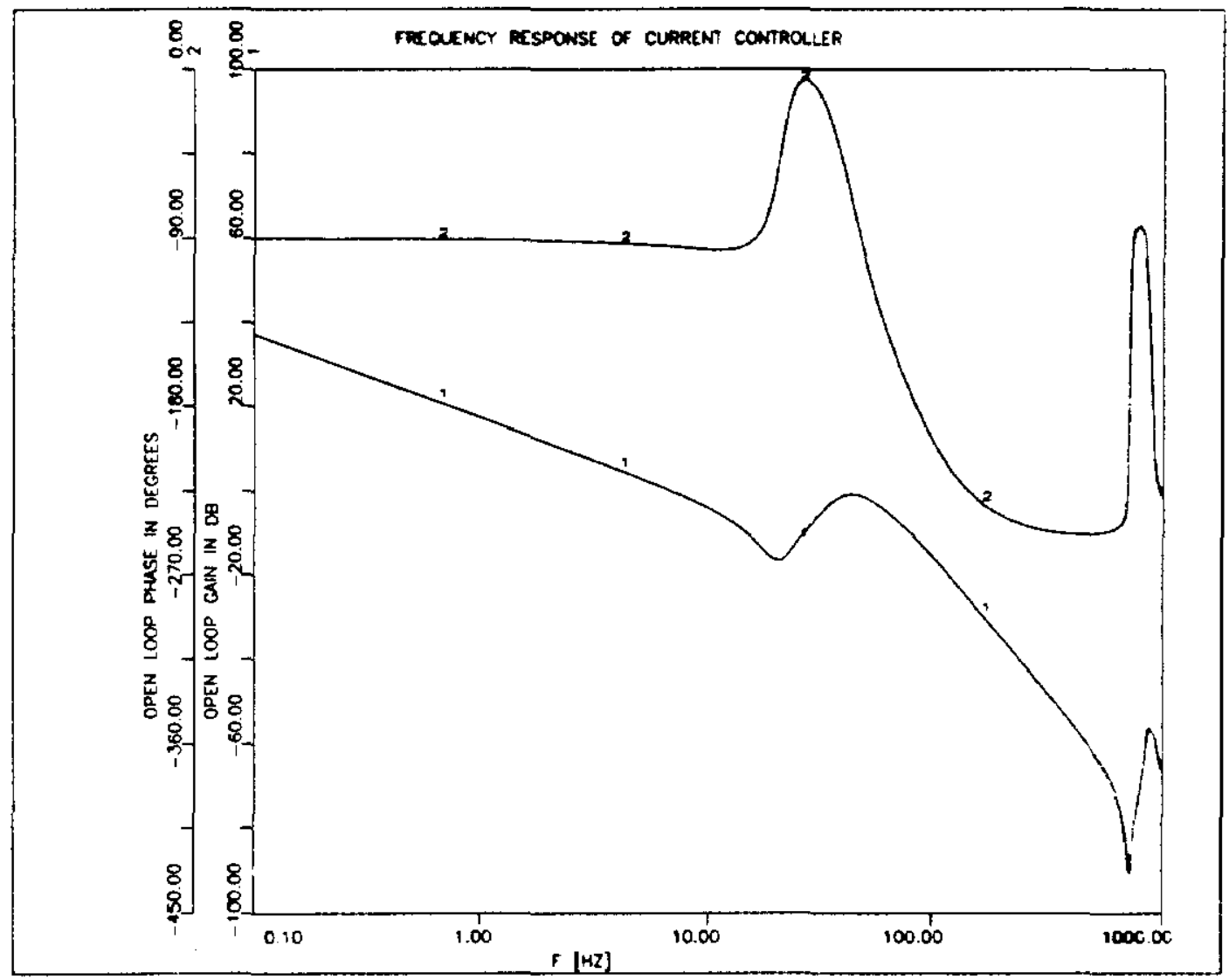

Figure 6. Frequency Response of Individual Sector Current Regulator.

Two kinds of perturbations were analyzed; a unit step change in the current reference while maintaining the voltage reference constant, and a $10 \mathrm{~V}$, $10 \mathrm{~ms}$ voltage pulse at the power supply output terminals. The responses to these perturbations are shown in figures 7 and 8 . The transient waveforms of the current show initial resonances determined by the transmission line characteristics. From the figure, the current rise time for the individual sectors can be determined for different values of the current controller gain. 


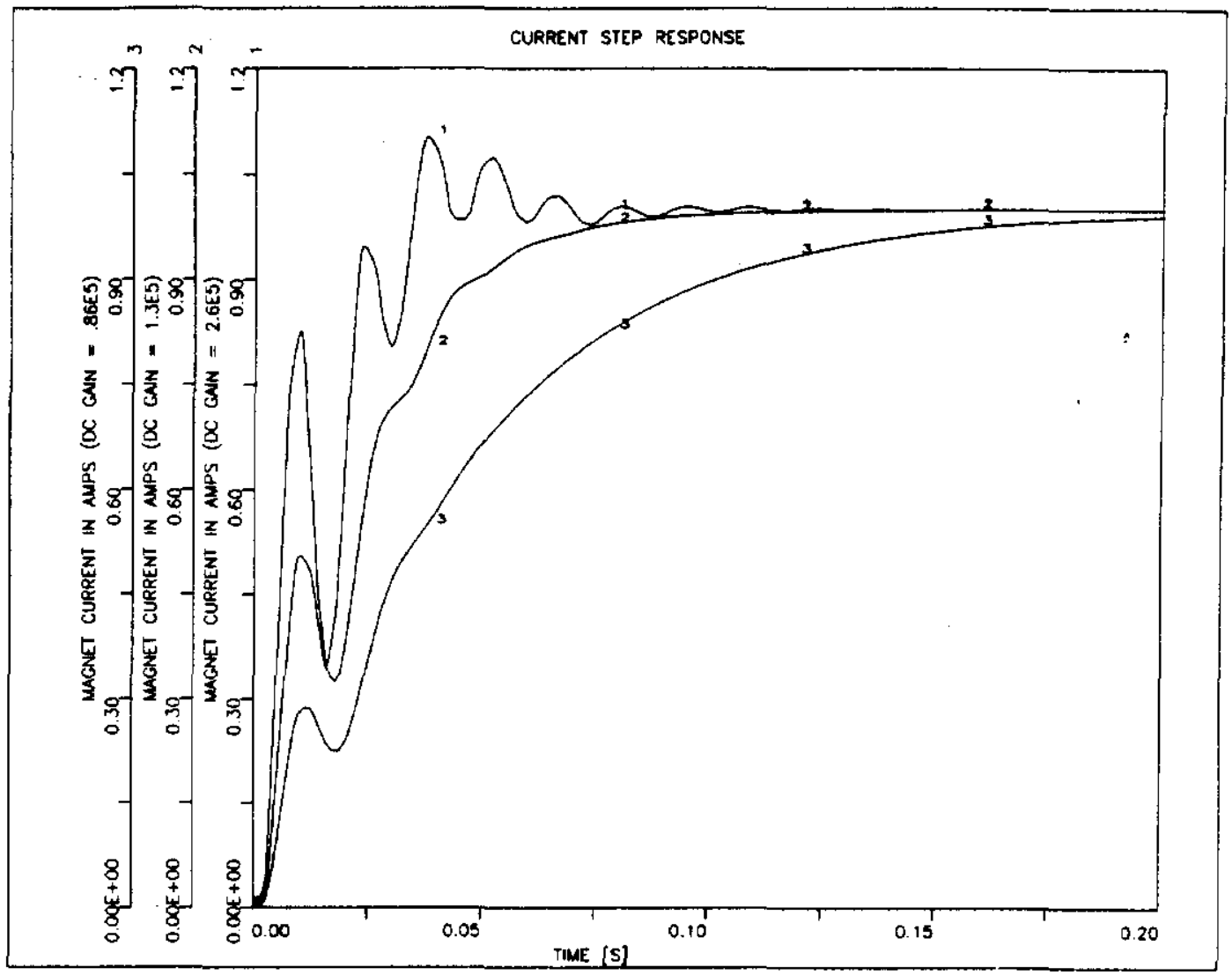

Figure 7. Transient Response to a Unit Step in the Current Reference for Different Gains in the Current Controller

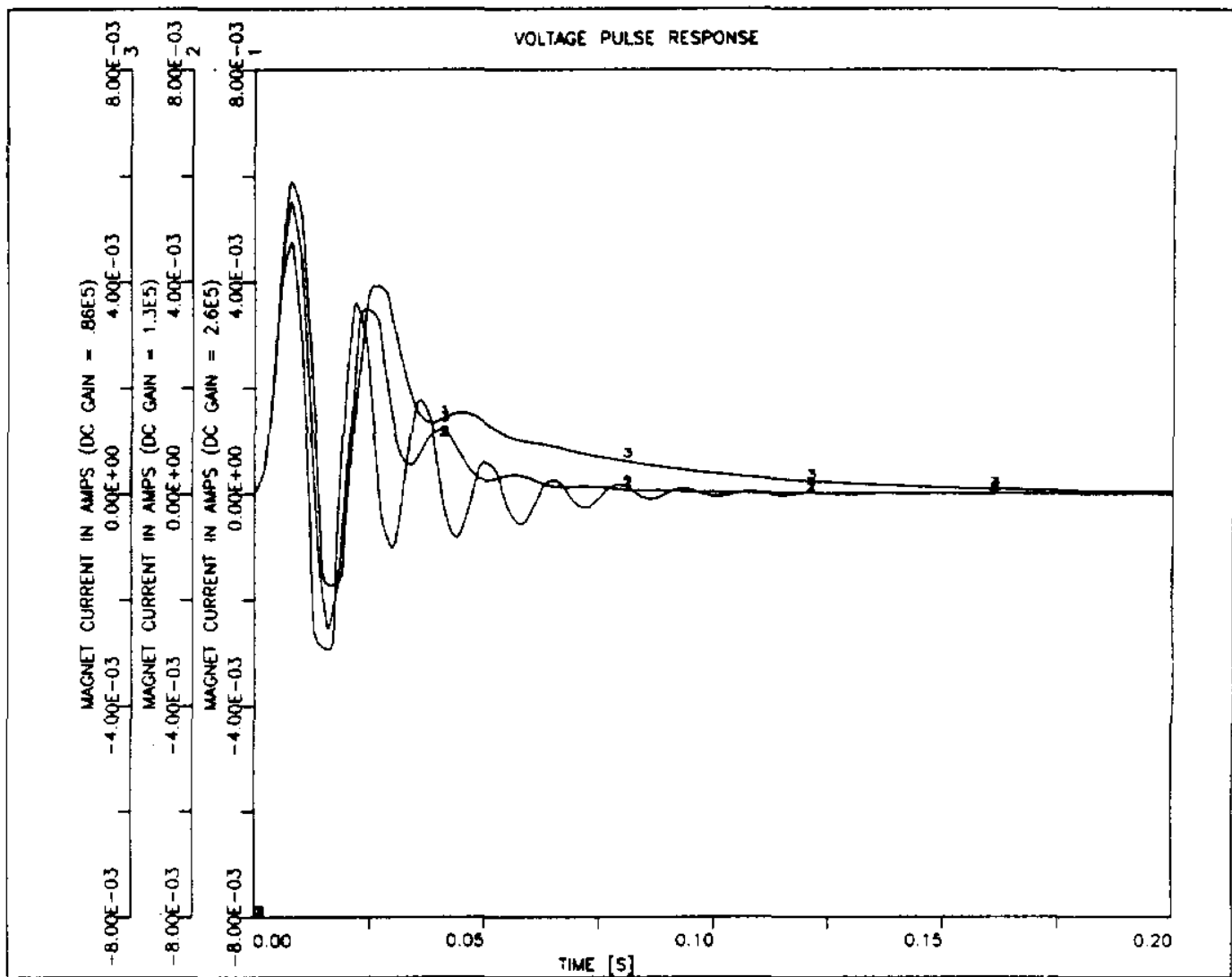

Figure 8. Transient Response to a $10 \mathrm{~V}, 10 \mathrm{~ms}$ Pulse Perturbation in the Voltage Power Supply for Different Current Controller Gains 


\section{Analysis of the Complete System}

As mentioned previously, the complete magnet system consists of ten sectors, identical to the one described before and electrically isolated. It is impossible to construct the ten loops to be identical. If all the circuits have the same voltage and current references, the regulated currents will be different. A complete simulation of all the error sources is a very difficult task and the final values have to be obtained making measurements on the real circuits, but we can estimate which error sources are dominant.

The main discrepancies considered here are caused by the initial ratio error of the current transductor of $\pm 100 \mathrm{ppm}$, offset of the transductor of \pm 10 $\mathrm{ppm}, \mathrm{D} / \mathrm{A}$ converters with $\$ 1.5 \mathrm{ppm} / \mathrm{C}$ temperature drift, $\neq 6 \mathrm{ppm}$ absolute accuracy and $\pm 2 \mathrm{ppm}$ noise. Among these errors, the initial error of the transductor gain dominates, and though it can be cancelled to some degree with initial adjustment for each sector, this is hard to do for all of them.

A possible control scheme that minimizes the errors associated with individual regulation loops in the isolated sectors working from the same reference, but different absolute transductors, utilizes one absolute transductor in the master sector and nine differential transductors measuring the differences between adjacent sectors.

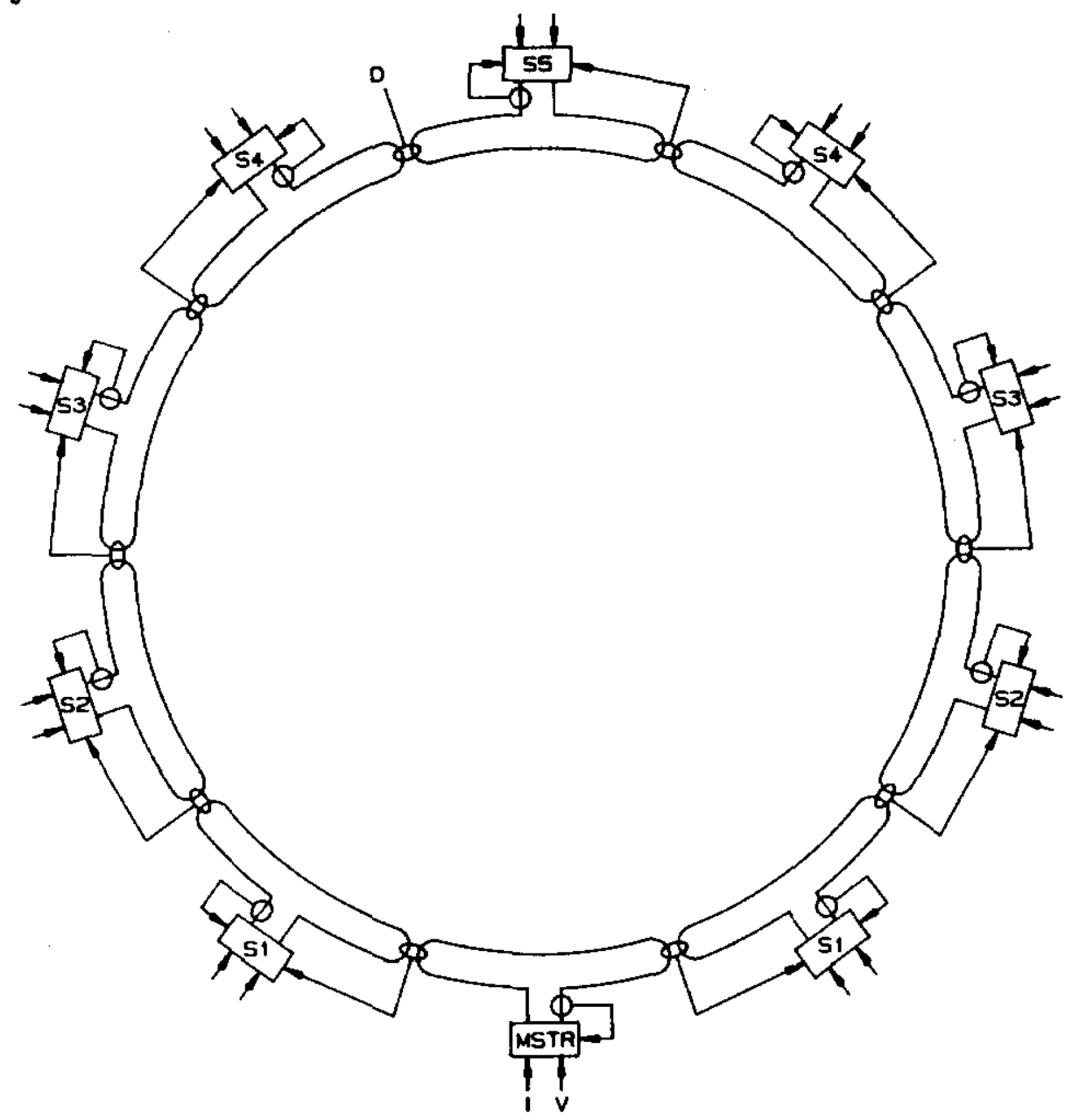

Figure 9. SSC Ring with Isolated Sectors and Differential Transductors 
If we use differential transductors to measure the difference between the currents in adjacent sectors, the full scale signals developed by these transductors represent a small fraction of the actual current. In this case, the tracking errors produced by the dispersion between the ratios in the different transductors is considered to be negligible The other error sources of the transductor and electronics, like scale factor, offset and noise are then dominant. Since the effect of these errors is related to the full scale value of the transductor used, its range must be determined. A compromise between improvement factor and linear range of operation suggests a value of $60 \mathrm{~A}$. Under this situation the total dc tracking error becomes less then $\neq 10 \mathrm{ppm}$ related to $6.6 \mathrm{kA}$.

A disadvantage of a system with a master following the reference and nine slaves tracking the master in a serial mode is that the system will be very slow leading to large errors during ramping. A combination of two types of transductors is desired; an absolute transductor in each cell following the reference in a fast loop and a differential transductor in a slow loop with high gain to minimize stationary errors. Such a scheme is shown in block diagram form in figure 9. An individual sector loop is shown in figure 10, where IREF represents the absolute current reference, VREF indicates the voltage reference to the power supplies and ID is a differential reference added to the circuit to allow special tests. and in this case set to zero.

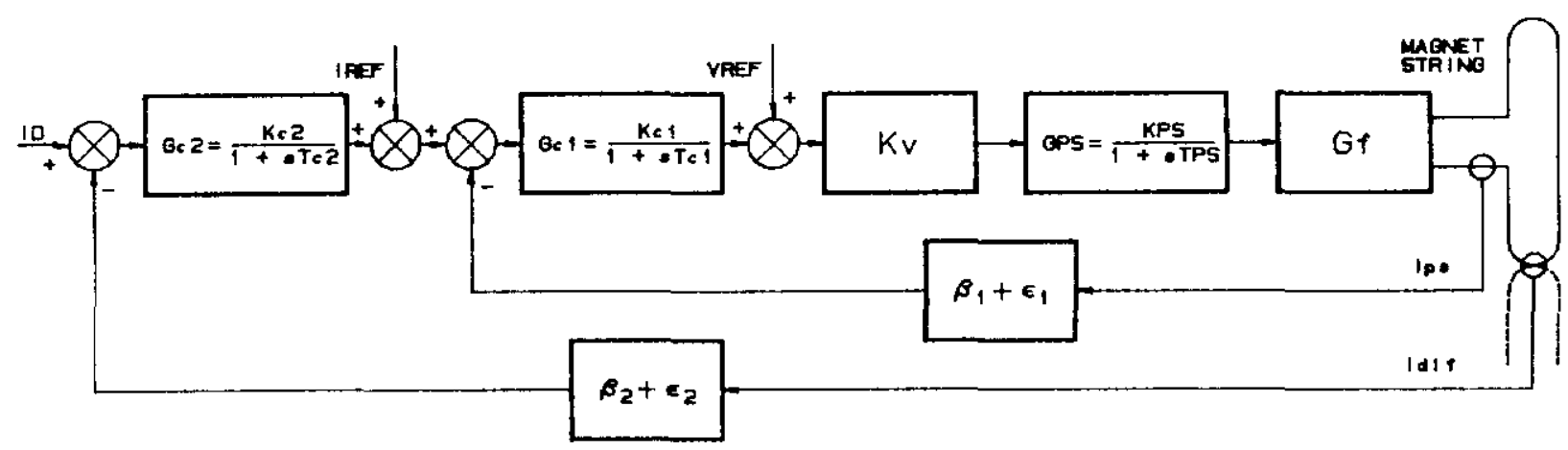

Figure 10. Regulator Block Diagram Including Differential Transductor Comparing Sectors

A low pass filter with a very long time constant was chosen for the differential loop controller as being the simplest circuit providing low gain at high frequencies while allowing the system to follow the reference and have high dc gain. Its gain was adjusted to maintain reasonable stability margins with fast response. The final offset from the desired value in the total current will be mainly òetermined by the errors in the master cell. In order to investigate the dynamic response of the master and slaves, a step was made in the master reference while holding the slave references constant. The result is shown in figure 11. 


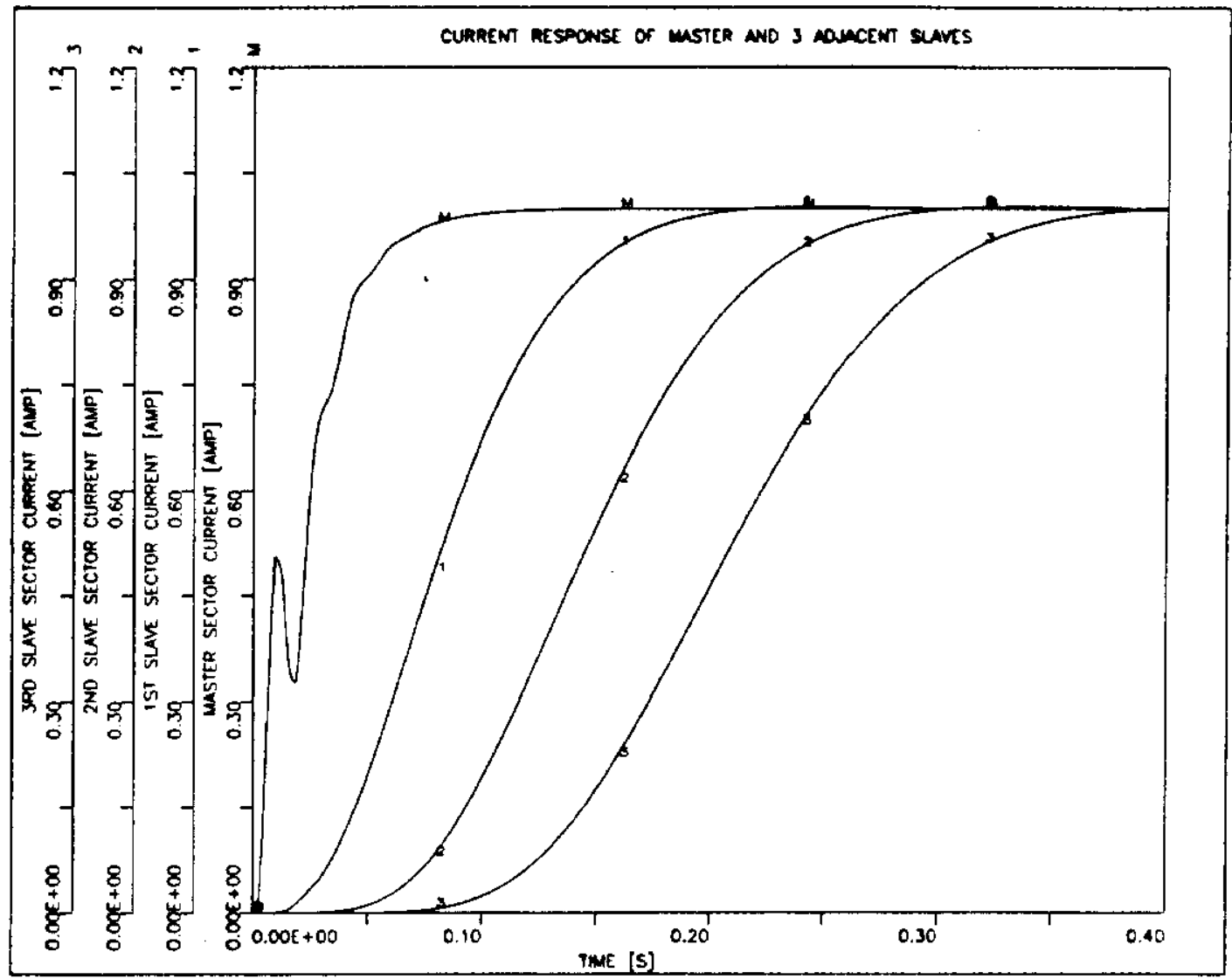

Figure 11. Transient Response of Master and Slave Sectors for a Step Change in the Master Current Reference.

As figure 11 shows, if a perturbation occurs in the master sector, the propagation delays accumulate along the ring. It would be desirable to minimize these accumulative delays.

The currents in the various slave sectors are:

$$
\begin{aligned}
& \mathrm{IS} 1=\mathrm{ISO}+\epsilon \mathrm{S} 0, \mathrm{SI} \\
& \mathrm{IS} 2=\mathrm{IS} 1+\epsilon \mathrm{S} 1, \mathrm{~S} 2 \\
& \text { IS5 }=\dot{\mathrm{IS} 4}+\epsilon \dot{\mathrm{S}} 4, \mathrm{~S} 5 \\
& \text { Where: } \text { SO }=\text { Master sector } \\
& \mathrm{Sn}=\text { Slave sector } \mathrm{n} \\
& \epsilon=\text { Error between adjacent sectors } \\
& \mathrm{ISn}=\mathrm{Absolute} \text { current in sector } \mathrm{n}
\end{aligned}
$$


Then

$$
\mathrm{ISn}=\mathrm{ISn}-1+\epsilon \mathrm{Sn}-1, \mathrm{Sn}=\mathrm{ISn}-2+\epsilon \mathrm{Sn}-2, \mathrm{Sn}-1+\epsilon \mathrm{Sn}-1, \mathrm{Sn}
$$

can be expressed as

$$
\mathrm{ISn}=\mathrm{IS0}+\sum \epsilon \mathrm{Si}-1, \mathrm{Si}+\epsilon \mathrm{Sn}-1, \mathrm{Sn}
$$

Since the goal of the regulation system is to match the individual sectors to the master sector, the most useful correction signal would be the difference between the master and slaves. As can be seen in the above expression, this is just the sum of all preceeding differential error signals including that of the present sector. Figure 12 shows a topology for implementing such a system.

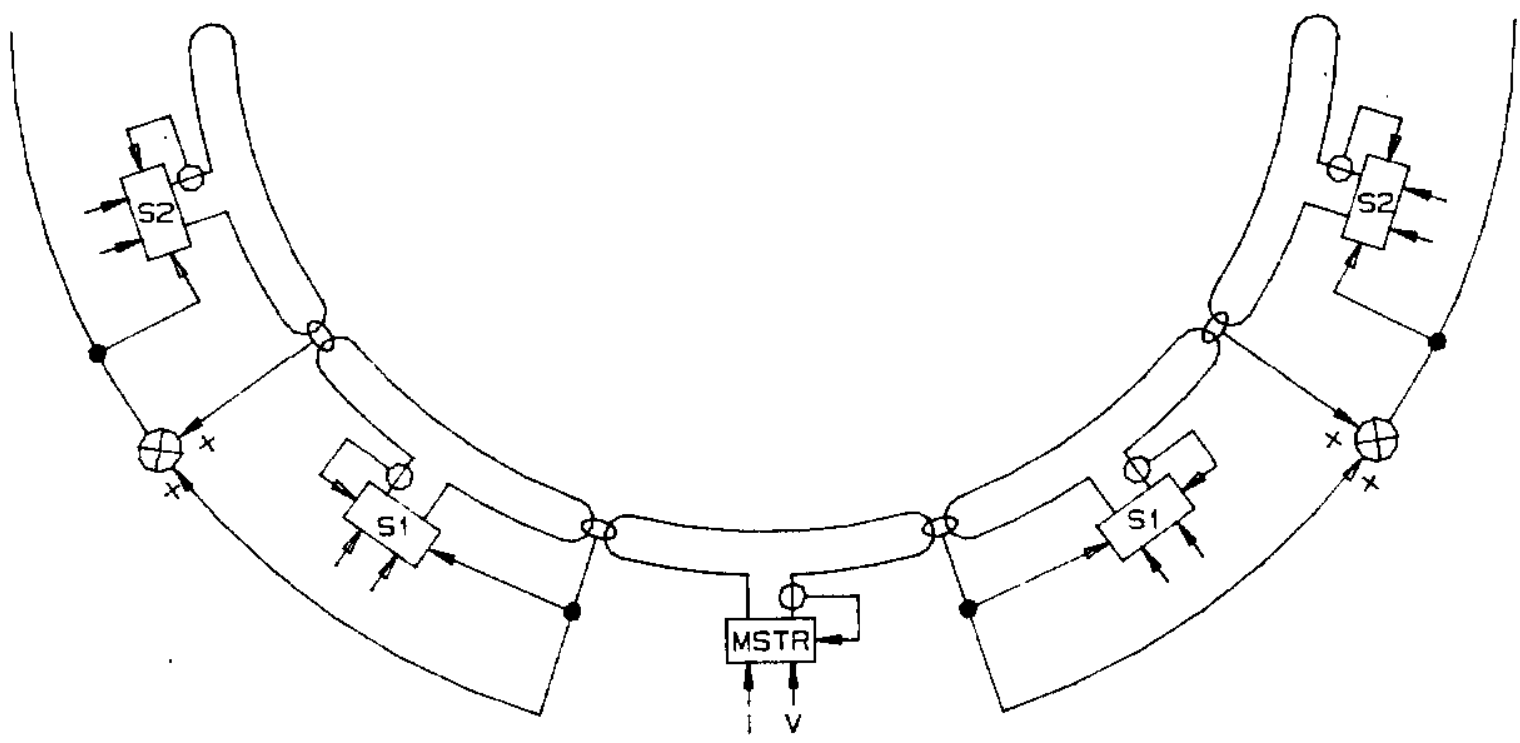

Figure 12. Improved SSC Ring Configuration

This correction technique was implemented in the SPICE model previously used. As figure 13 shows, the effect of this change is to provide every slave with a reference that is the equivalent of the master current. All slaves now have an identical response delay from the master that is dependent only on the delay through the master sector magnet string. The stability of the system is not affected by this change. 


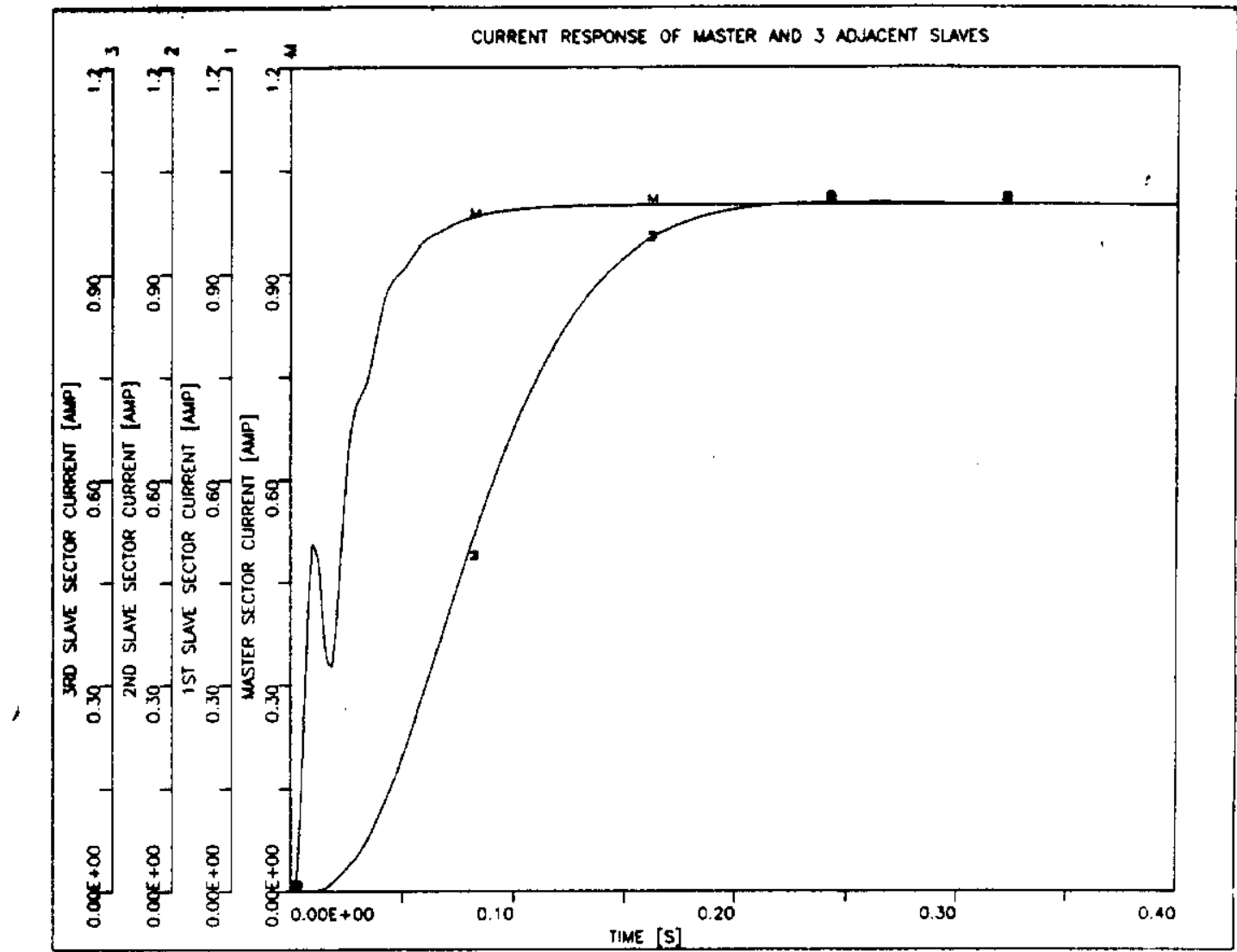

Figure 13. Transient Response of Master and Slave Sectors With Improved Compensation

\section{Conclusions}

This first analysis of the stability and overall regulation of the system indicates the convenience of having differential transductors in order to maintain a low dispersion between all the magnet sector currents. The analysis shows that, since the differential loop signals are propagated in a cascade mode (without feeding back to previous sectors) the stability of the individual sectors will guarantee the overall stability of the system. It is also noted that the delays produced by the transmission line characteristics of the magnets have no serious effect on the differential loop response because they are too small compared with the time constant of this loop.

The addition of the previous stage error in the improved compensation scheme is also recommended. Its main advantage is to improve the transient response of the slaves to changes in the master current.

Finally, the whole differential scheme, including the correction techniques, is felt to be relatively simple in terms of hardware and design effort. 